\title{
Localization and Damage Induced Softening using Finite Element and Smooth Particle Hydrodynamic Methods
}

\author{
N. Djordjevic ${ }^{1, *}$, R. Vignjevic ${ }^{1}$, T. De Vuyst ${ }^{1}$, S. Gemkow ${ }^{2}$, J. Campbell ${ }^{1}$, K. Hughes ${ }^{1}$ \\ ${ }^{1}$ Dynamic Response Group, Structural Integrity Theme, Brunel University London, Kingston \\ Lane Uxbridge, UB8 3PH, United Kingdom \\ e-mail: nenad.djordjevic@brunel.ac.uk; v.rade@brunel.ac.uk \\ ${ }^{2}$ Cranfield University, Cranfield, Bedfordshire MK43 0AL, United Kingdom \\ *corresponding author
}

\begin{abstract}
The main aim of this work is investigation of localization problem in strain softening materials and regularization techniques, which will reduce and possibly remove mesh dependency of the numerical results and balance the effects of heterogeneous microstructure on local continua while keeping the boundary value problem of softening (damaged) continua well-posed. Finite Element Method (FEM) and Smooth Particle Hydrodynamic (SPH) combined with a local continuum damage model (CDM) were used for analysis of a dynamic stress wave propagation problem, which was analytically solved in (Bažant and Belytschko 1985). The analytical solution was compared to the numerical results, obtained by using a stable, Total-Lagrange form of SPH (Vignjevic et al. 2006, Vignjevic et al. 2009), and two material models implemented in the FEM based on: 1) classic CDM; and 2) equivalent damage force. The numerical results demonstrate that the size of the damaged zone is controlled by element size in classic FEM and the smoothing length in the SPH, which suggests that the SPH method is inherently non-local method and that the smoothing length should be linked to the material characteristic length scale in solid mechanics simulations.
\end{abstract}

Keywords: continuum damage, strain softening, localization, SPH, FEM

\section{Introduction}

When material response to the mechanical loading includes degeneration of material properties, application of the local continuum damage mechanics (CDM) models to the strain softening materials, including some composites, is rather limited. In this case, a local damage model implemented in the finite element method (FEM) leads to an ill posed boundary value problem, where the local governing hyperbolic differential equations at a point become elliptic, which leads to numerical instability. This instability is mesh-sensitive and manifests itself as nonphysical deformation of the softening continuum (due to deformation localization, infinite number of bifurcated branches and post-bifurcation mesh dependency issues).

A well-known solution to the localization problem is addition of a characteristic length scale to CDM models, a non-local approach, which maintains the character of the governing equations in the material softening deformation regime. The characteristic length scale is typically defined 
either in the form of spatial gradients (Dillon and Kratochvill 1970, Aifantis 1984, Bammann and Solanki 2010) or integral nonlocal terms, see for instance (Pijaudier-Cabot and Bazant 1987, Tvergaard and Needleman 1995, Tvergaard and Needleman 1997, Enakoutsa et al. 2007). Non-local approach based on the introduction of length scale provides a framework for modelling relevant aspects of the underlying physics (sub continuum scale effects) of materials.

Two discretization methods were considered in this work: FEM and Smoothed Particle Hydrodynamics (SPH) (Gingold and Monaghan 1977, Libersky and Petschek 1991, Libersky et al. 1993). The SPH is a meshless particle method, which does not require a structured grid. The motion of the continuum is approximated by motion of discrete material points (particles) with no fixed connectivity. Interaction of particles is defined by a weighting (smoothing) function, where the smoothing length (size of the smoothing function domain) defines the range of influence of an individual particle. It is this smoothing length scale that makes the SPH a nonlocal method.

The main aim of this paper was the analysis of strain softening effects observed in a dynamic stress wave propagation problem using FEM and SPH method, with two specific objectives: 1) to establish if SPH is by nature a nonlocal method capable of overcoming difficulties related to material softening without any additional regularization measures; and 2) to find a suitable solution for the localization problem observed in the classic FEM. The chosen one-dimensional stress wave propagation problem, analytically solved in (Bažant and Belytschko 1985), was modelled in LLNL Dyna3D and in house developed SPH code. The exact analytical solution was compared to the numerical results, obtained by using a stable, Total-Lagrange form of SPH described in (Vignjevic et al. 2006, Vignjevic et al. 2009), and two models implemented in the explicit FEM. The first material model was developed using classical continuum damage mechanics approach, whilst the second model was a proposed solution for strain softening problem, developed based on equivalent damage force.

This paper consists of four sections: following the introduction on the dynamic strain softening problem given above, analytical solution of the problem is given in Section 2, which is followed by the numerical experiments described in Section 3. Conclusion and future work are presented in Section 4.

\section{Dynamic strain softening problem and its analytical solution}

One dimensional stress wave propagation problem defined in (Bažant and Belytschko 1985) is shown in Fig. 1. The problem is $2 L$ long bar, symmetrically loaded at both ends with a constant velocity $v$. In the original paper (Bažant and Belytschko 1985), material behavior of the bar was determined by stress strain relationship illustrated in Fig. 1b, where the softening zone between Point $\mathrm{P}$ and Point $\mathrm{F}$, is characterized with a negative slope and elastic unloading/reloading law.

The loading defined in Fig. 1 generates two tensile step stress waves, which propagate towards the midsection of the bar $(x=0)$, where they are superposed at time $t=L / c$. The Superposition of the strain waves in the midsection of the bar instantaneously doubles the strain at that point, which can result in strain softening material behavior. 

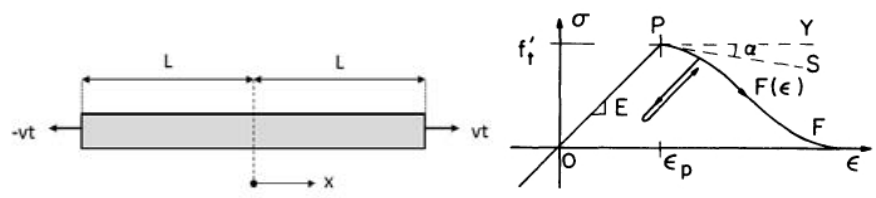

Fig. 1. (a) Geometry and loading of softening bar; (b) stress strain law (Bažant and Belytschko 1985)

The analytical solution for this stress wave propagation problem was defined with the following set of equations for displacement, strain and stress components in the loading direction:

$$
\begin{gathered}
\varepsilon_{x}=\frac{\partial u}{\partial x}=\frac{v}{c}\left[H\left(t-\frac{x+L}{c}\right)+H\left(t+\frac{x-L}{c}\right)\right] \\
u(x, t)=-v\left\langle t-\frac{x+L}{c}\right\rangle+v\left\langle t+\frac{x-L}{c}\right\rangle \\
\sigma_{x}=\frac{E(1-v)}{(1-2 v)(1+v)} \varepsilon
\end{gathered}
$$

Where: $u(x, t)$ is displacement, $\varepsilon_{x}$ strain in loading $x$ direction, $\sigma_{x}$ stress in loading $x$ direction, $H$ is Heaviside function, $t$ is time, $c$ is elastic speed of sound and $E$ and $v$ are Young's modulus and Poisson's ratio, respectively.

The analytical solutions for displacement, strain, stress and internal energy in the strain softening problem, at the response time $t=3 L / 2 c$, are shown in Fig. 2. The key difference between the elastic nonlocal solution and the strain softening (local) solution is discontinuity in the displacements and development of the standing strain wave in the midsection of the bar, which occurred as a result of superposition of the waves propagating from the bar ends. The obtained discontinuity could not propagate away from the localization zone, so that material unloads outside of the localization zone. In other words, the softening zone acts as a free boundary.

\section{Simulation results for dynamic strain softening problem}

The dynamic strain softening problem described in the previous section was modelled by using two models implemented in the FEM and a classic CDM model implemented in the SPH, with the material behavior described as a bilinear constitutive law with isotropic material properties. Following an overview on the SPH method and the implemented constitutive law, the numerical results are presented in three subsections, starting from the classic FEM, followed by SPH solution and a nonlocal equivalent damage force (EDF) method. 
a)
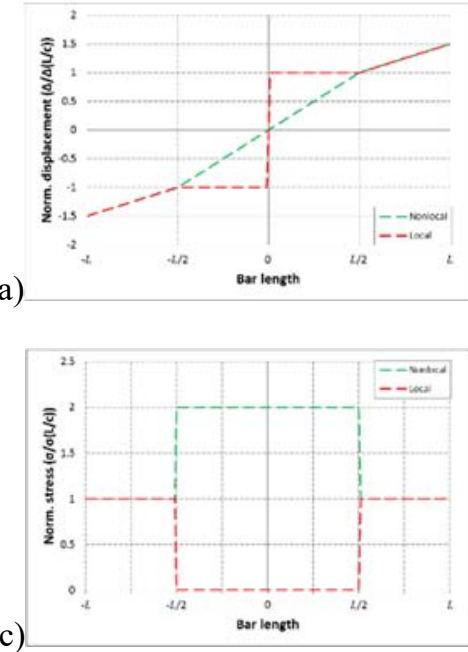

b)
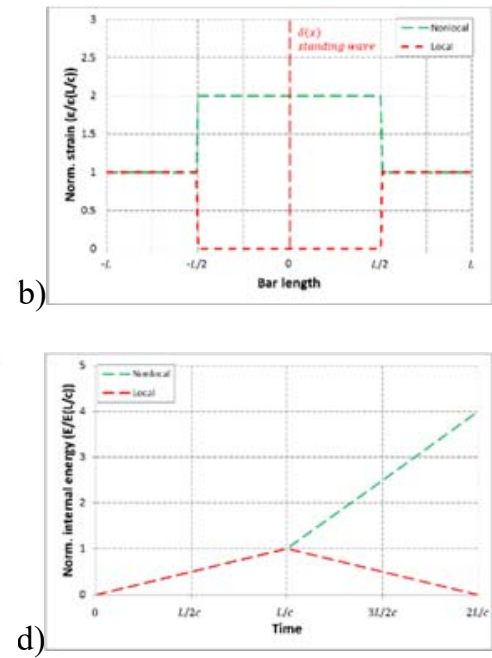

Fig. 2. Elastic local and nonlocal solutions at the time instance $t=3 \mathrm{~L} / 2 \mathrm{c}$ for: (a) Normalised displacement; (b) Normalised strain; (c) Normalised stress; (d) Normalised internal energy

SPH is a meshless particle method which does not require a structured grid. The motion of the continuum is approximated by the motion of discrete material points (particles) with no fixed connectivity. Interaction of particles is defined by a weighting (smoothing) function, where the smoothing length (size of the smoothing function domain) defines the range of influence of an individual particle. For instance, an estimate value of the function $f(\boldsymbol{x})$ at the location $\boldsymbol{x}$ is given as a continuous integral function in the following form:

$$
\langle f(\boldsymbol{x})\rangle=\int_{\Omega} f\left(\boldsymbol{x}^{\prime}\right) W\left(\left|\boldsymbol{x}-\boldsymbol{x}^{\prime}\right|, h\right) d \boldsymbol{x}^{\prime}
$$

where the angled brackets $\langle\cdot\rangle$ denote kernel approximation, $W$ is a weighting function, $\boldsymbol{x}^{\prime}$ is a new independent variable and $h$ denotes smoothing length, i.e. size of the kernel support. The smoothing length is illustrated in Fig. 3 and calculated from inter-particle distance, $p$, as:

$$
h=\lambda \Delta p
$$

A discrete form of the kernel approximation, where the function is known at a finite number of points $N$, is given in terms of a sum:

$$
\left\langle f\left(\boldsymbol{x}_{I}\right)\right\rangle \approx \sum_{J} \frac{m_{J}}{\rho_{J}} f\left(\boldsymbol{x}_{J}\right)\left[W\left(\left|\boldsymbol{x}_{I}-\boldsymbol{x}_{J}\right|, h\right)\right]
$$

with the corresponding gradient equal to:

$$
\nabla f\left(\boldsymbol{x}_{I}\right)=\sum_{J} \frac{m_{J}}{\rho_{J}} f\left(\boldsymbol{x}_{J}\right)\left[\nabla W\left(\left|\boldsymbol{x}_{I}-\boldsymbol{x}_{J}\right|, h\right)\right]
$$

For more information on the in house developed SPH code available at Brunel University London, please see (Vignjevic et al. 2006, Vignjevic et al. 2009). A specific objective of this 
investigation was to establish if SPH is by nature a nonlocal method capable of overcoming difficulties related to material softening without any additional regularization measures.

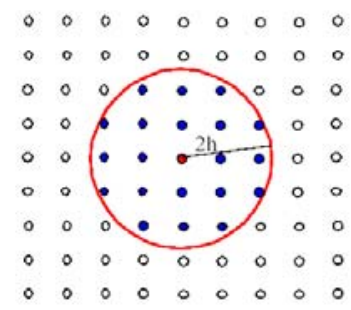

Fig. 3. Definition of neighborhood in SPH discretization method

Stress strain relationship of an isotropic bilinear material model with damage, implemented in the two numerical codes, is shown in Fig. 4. The damage was described with a single damage parameter $\omega$, using a local continuum damage mechanics approach. The strain softening and the damage evolution developed when strain was in a range between $\varepsilon_{i}$ and $\mathrm{e}_{f}$.

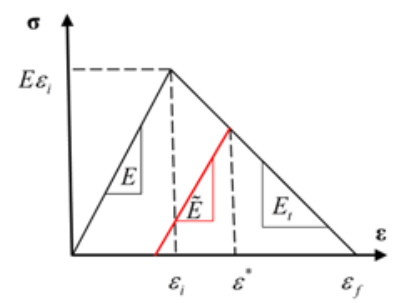

Fig. 4. Bilinear law implemented in the FEM and SPH codes using a damage parameter $\omega$ and classic CDM approach

The effective stress, damage and tangent stiffness/slope of the stress stain curve at the material state determined by $\varepsilon^{*}$, were respectively calculated as:

$$
\begin{gathered}
\bar{\sigma}=\frac{\sigma}{1-\omega} \\
\omega=1-\frac{\varepsilon_{i}\left(\varepsilon_{f}-\varepsilon^{*}\right)}{\varepsilon^{*}\left(\varepsilon_{f}-\varepsilon_{i}\right)} \\
E_{T}=-\frac{E \varepsilon_{i}}{\varepsilon_{f}-\varepsilon_{i}}
\end{gathered}
$$

In the equations above, $\sigma$ and $\bar{\sigma}$ are, true stress and effective stress respectively, whist $E$ is a Young modulus of the virgin material.

The experimental programme consisted of the simulations run with four FEM and three SPH models with different discretion densities as shown in Fig. 5 and Fig. 6. 


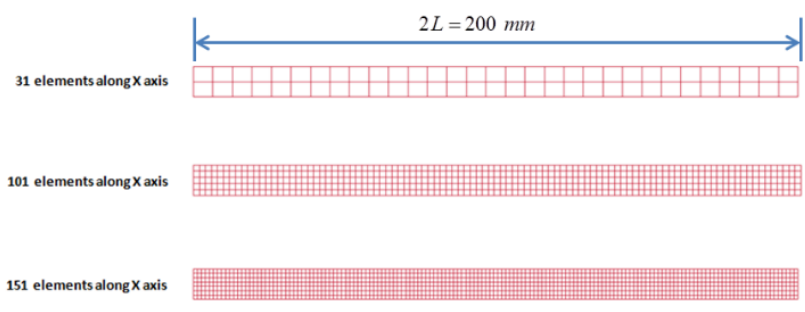

201 elements along $X$ axis

Fig. 5. Spatial discretization used in the FEM simulations of the dynamic strain-softening bar

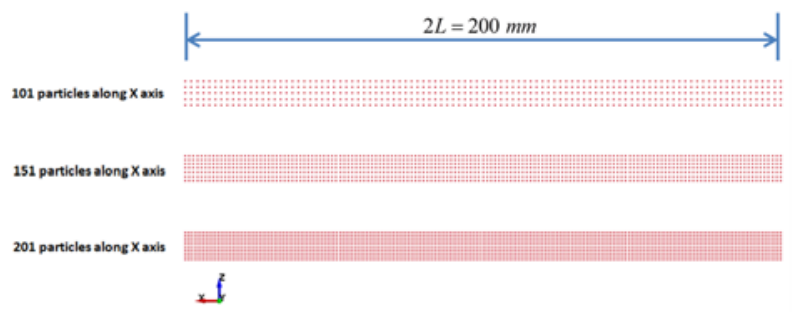

Fig. 6 Particle discretization in SPH of the dynamic strain-softening bar

\subsection{Classic FEM analysis of strain softening problem}

The numerical results obtained with four FEM models combined with classic local CDM damage formulation are shown in Fig. 7 and compared with the local analytical solution for displacement, strain and stress field, at response time $t=3 L / 2 c$. The results show a strong dependence on the mesh density in the strain-softening area as a consequence of the local strain-softening. The numerical results are converging to the local analytical solution with the increase in the mesh density. The strain developed in a single element in the midsection of the bar and its magnitude increased with the mesh refinement i.e. reduction of the size of the softening zone. This strain increase in the midsection of the bar led to the rapid development of damage parameter to the value of 1 , which corresponds to total failure. The damaged zone was limited to a single element and the size of the softening zone in which the damage accumulates was influenced by the initial element size, as illustrated in Fig. 8. 
(a)
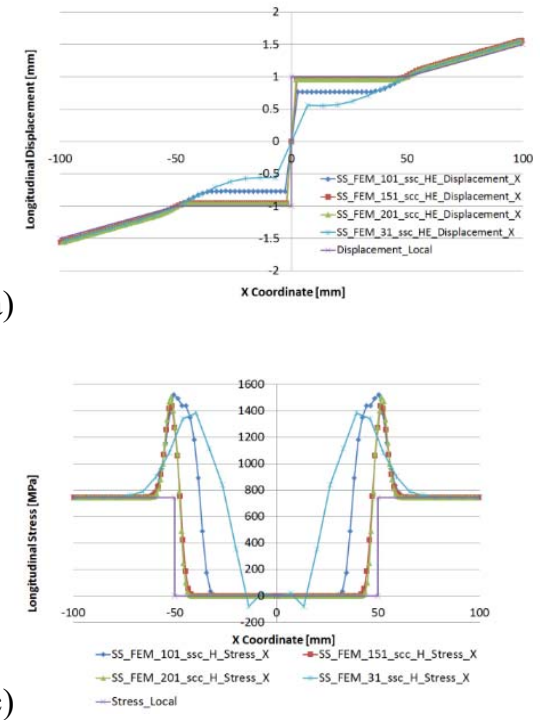

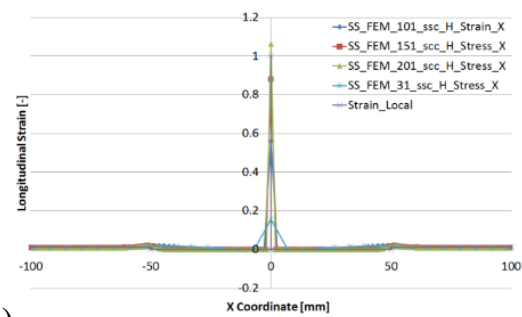

(b)

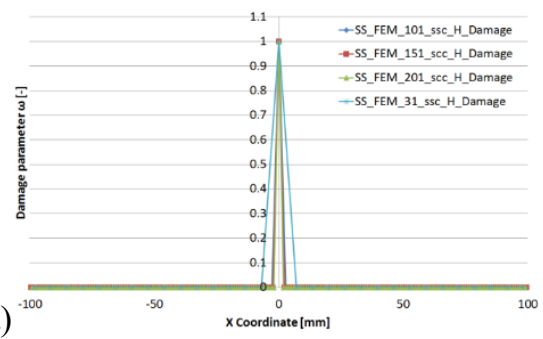

Fig. 7. Classic FEM solutions of dynamic strain softening problem at the time instance $t=3 \mathrm{~L} / 2 \mathrm{c}$ for: (a) Longitudinal displacement; (b) Longitudinal strain; (c) Longitudinal stress; (d) Damage distribution

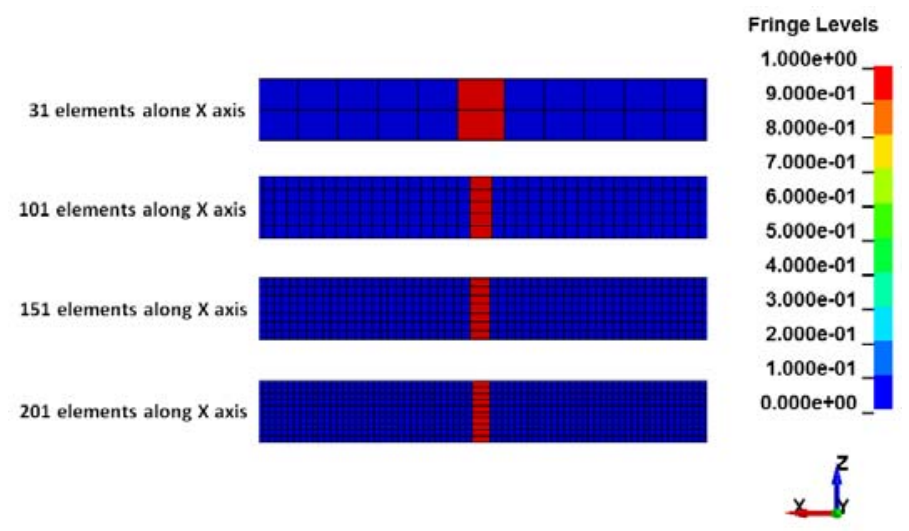

Fig. 8. FEM results for damage distribution within dynamic strain softening problem using classical CDM approach; (fringe level: damage [-])

One possible way to address this problem is alternative approach to modelling damage described in the Subsection 3.3.

\subsection{SPH analysis of strain softening problem}

The SPH simulation programme was run with three discretization densities shown in Fig. 6. The influence of the smoothing domain on the numerical results was analyzed in terms of each parameter that constitute the smoothing length. Consequently, three sets of numerical tests were run with three discretization densities for analysis the influence of: 1) inter-particle distance $\Delta p$ ; 2) parameter $\lambda$; and 3 ) smoothing length, $h$. It was observed that the SPH method is 
inherently nonlocal and that the results were sensitive to the changes of the smoothing length. However, when the smoothing length was kept constant between the three models with different discretization densities (interparticle distance $\Delta p$ ), the numerical results for displacement, strain, stress and damage remained the same as illustrated in Fig. 9. The damage distribution obtained with three models with the fixed smoothing length shown in Fig. 10 is independent of the interparticle distance $h$; instead the damage zone within the three models was constant and equal to $4 h$, which suggests that the smoothing length should be considered as a material property - characteristic length.

(a)

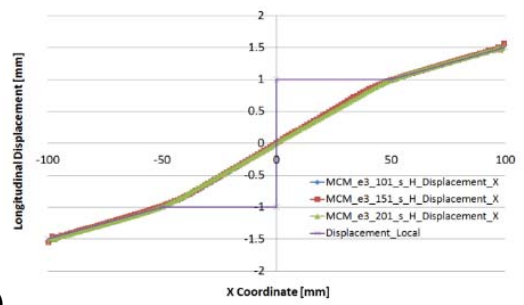

(b)

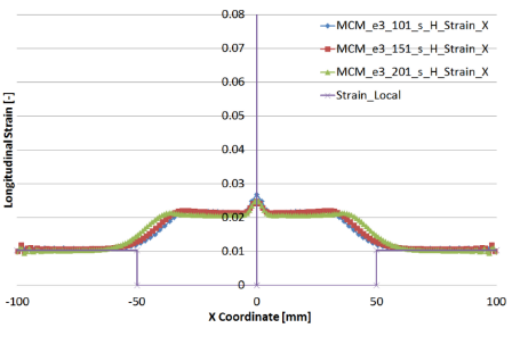

(d)

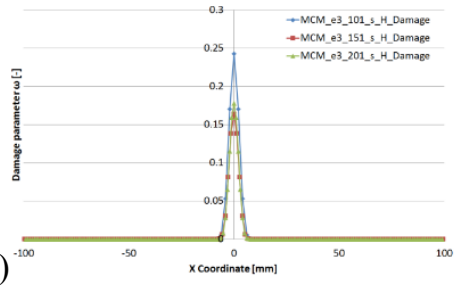

(c)

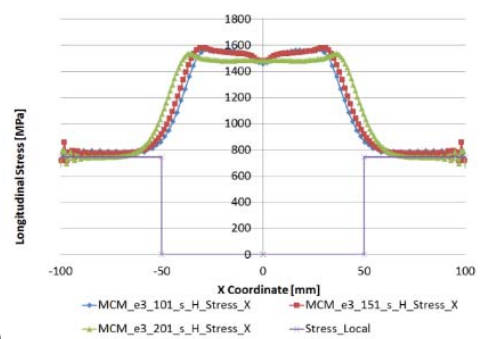

Fig. 9. $\mathrm{SPH}$ solutions of dynamic strain softening problem at the time instance $t=3 \mathrm{~L} / 2 \mathrm{c}$ for: (a) Longitudinal displacement; (b) Longitudinal strain; (c) Longitudinal stress; (d) Damage distribution

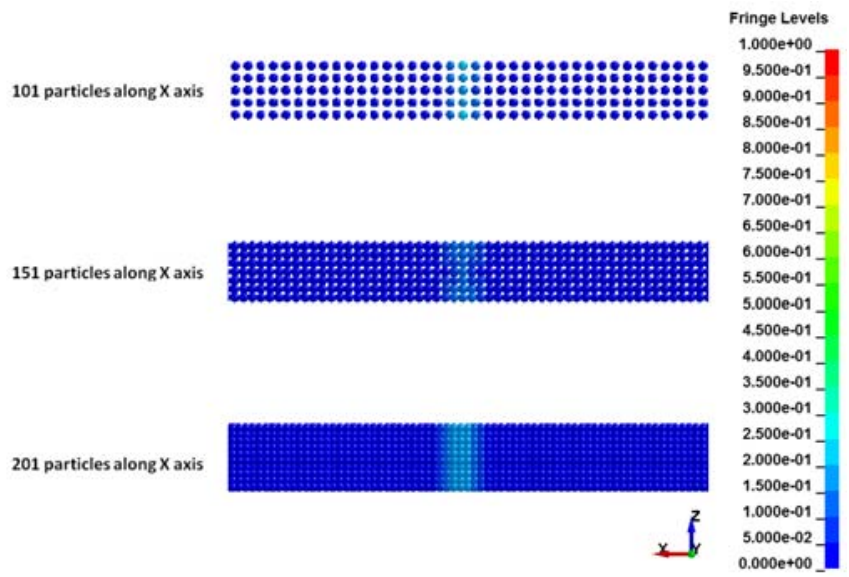

Fig. 10. Localization of damage within a limited zone $4 \mathrm{~h}$ in size $(\mathrm{h}=2.5 \mathrm{~mm})$ around the bar symmetry plane at response time $\mathrm{t}=3 \mathrm{~L} / 2 \mathrm{c}$ (fringe level: damage $[-]$ ) 


\subsection{FEM analysis of strain softening problem with EDF}

The first attempt to address the pronounced localization problem of the classic FEM was made by using an equivalent damage force as an alternative method for modelling damage, where the damage contribution to the conservation of momentum was defined by the means of divergence of damage and stress tensors. This approach did not affect the well posiness of the boundary problem, provided the nonlocal solutions for damage distribution in the considered wave propagation problem and allowed for implementation of the material characteristic length scale in the FEM solution. The first implementation of the equivalent damage force model was done in in-house developed FEM/SPH code and the numerical experiments were run for a onedimensional wave propagation problem described above. The simulation results for damage distribution equivalent to the results shown in Fig. 8 and Fig. 10, now obtained with the EDF model are shown in Fig. 11. The EDF results for damage distribution in the problem considered, are not localized in one layer of elements, but distributed over a limited zone which is approximately $3 l_{\omega}$ wide, where the $l_{\omega}$ is input parameter. The EDF results for displacement, strain and stress are stable and consistent with the nonlocal analytical solutions presented in Fig. 2 .

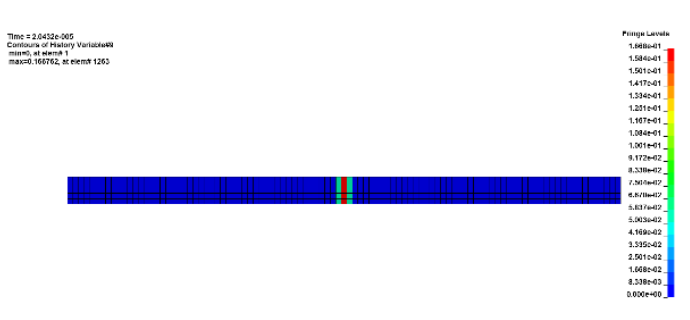

a)

L.

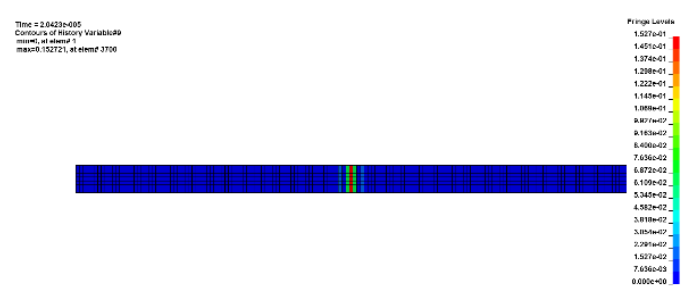

b)

$L^{x}$

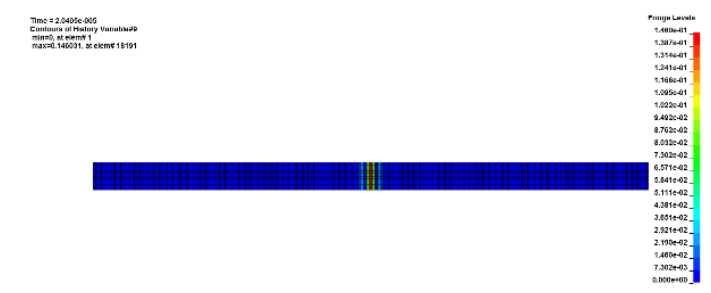

c)

Fig. 11. Finite element results for damage distribution within dynamic strain softening problem obtained with EDF model at response time $t=3 \mathrm{~L} / 2 \mathrm{c}$ (fringe level: damage [-]) with: (a) 101 element in impact direction; (b) 151 element in the impact direction; (c) 201 element in the impact direction 


\section{Conclusions}

A series of numerical experiments, using both SPH and FEM solvers, demonstrated that the width of the strain softening region is controlled by the element size in classic FEM but in SPH it is controlled by the smoothing length, rather than the inter-particle distance, which is analogous to the element size in the FEM. This demonstrates that the SPH method is inherently non-local and suggests that the SPH smoothing length should be linked to the material characteristic length scale in solid mechanics simulations. The first attempt to address the localization problem in the FEM model was made by alternative definition of damage effects using EDF. The numerical results obtained with this model show stable and nonlocal character, with a reduced mesh dependency, where the size of the damaged zone was controlled with characteristic length, as a material input parameter. Future work on this problem will be focused on extension of the equivalent damage force approach to orthotropic material formulation, suitable for composite materials.

Acknowledgements The project leading to this publication has received funding from the European Union's Horizon 2020 research and innovation programme under grant agreement No 636549.

\section{References}

Aifantis EC (1984). On the Microstructural Origin of Certain Inelastic Models, Journal of Engng Mater. Tech., 106, 326-334.

Bammann DJ, Solanki K (2010). On Kinematic, Thermodynamic and Coupling of a Damage Theory for Polycrystalline Material, International Journal of Plasticity, 26 6, 775-793.

Bazant ZP and Belytschko TB (1985). Wave Propagation In A Strain- Softening Bar: Exact Solution, Journal of Engineering Mechanics, 111, 381-389.

Dillon OW and Kratochvill J, (1970). A Strain Gradient Theory of Plasticity, International Journal of Solids and Structures, 6, 1533-1566.

Enakoutsa K, Leblond JB and Perrin G (2007). Numerical Implementation and Assessment of a Phenomenological Nonlocal Model of Ductile Rupture, Comput. Meth. Appl. Mech. Engng., 196, 1946-1957.

Gingold RA and Monaghan JJ (1977). Smoothed particle hydrodynamics, Theory and application to non-spherical stars, Mon Not R Astron Soc, 181, 375-389.

Libersky LD and Petschek AG (1991), Smooth particle hydrodynamics with strength of materials, Advances in the Free-Lagrange Method Including Contributions on Adaptive Gridding and the Smooth Particle Hydrodynamics Method (Eds. Trease HE, Fritts MJ and Crowley WP), USA, Springer, 248-257.

Libersky LD, Petschek AG, Carney TC, Hipp JR and Allahdadi FA (1993). High strain lagrangian hydrodynamics a three-dimensional SPH code for dynamic material response, $J$. Comput Phys, 109, 67-75.

Pijaudier-Cabot G and Bazant ZP (1987). Nonlocal Damage Theory, ASCE J. Engrg. Mech., $113,1512-1533$.

Tvergaard V and Needleman A (1995). Effects of Nonlocal Damage in Porous Plastic Solids, Int. J. Solids Struct., 32, 1063-1077.

Tvergaard V and Needleman A (1997). Nonlocal Effects on Localization in a Void-sheet, Int. J. Solids and Struct., 34, 2221-2238.

Vignjevic R Reveles JR and Campbell J (2006). SPH in a total Lagrangian formalism, CMESComp Model Eng; 14 (3), 181-198.

Vignjevic R Campbell J Jaric and J Powel S (2009). Derivation of SPH Equations in a Moving Referential Coordinate System, Comput Methods Appl. Mech. Engrg., 198, 2403-2411. 